\title{
On the strongly annihilating-submodule graph of a module
}

\author{
R. Beyranvand*(i), A. Farzi-Safarabadi (D) \\ Department of Mathematics, Lorestan University, Khorramabad, Iran
}

\begin{abstract}
In this paper we continue to study the strongly annihilating-submodule graph. In addition to providing the more properties of this graph, we compare extensively the properties of this graph with the annihilating-submodule graph.
\end{abstract}

Mathematics Subject Classification (2020). 05C78, 16D10, 13C13, 13A99

Keywords. annihilating-submodule graph, strongly annihilating-submodule graph, dominating number, girth, bipartite graph

\section{Introduction}

Throughout this paper $R$ is a commutative ring with nonzero identity element and $M$ is a unitary right $R$-module. For a submodule $N$ of $M$, denoted by $N \leq M$, the ideal $\{r \in R \mid M r \subseteq N\}$ will be denoted by $(N: R M)$ (briefly by $(N: M)$ ). Recall that $M$ is indecomposable if it is nonzero and cannot be written as a direct sum of two nonzero submodules. $M$ is called uniform if the intersection of any two nonzero submodules is nonzero. Also a submodule $N$ of $M$ is called an essential submodule of $M$, denoted by $N \leq_{e} M$, if for any nonzero submodule $K$ of $M, K \cap N \neq 0$. For $X \subseteq M$, the annihilator of $X$ in $R$ is the ideal $\operatorname{ann}_{R}(X)=\{r \in R \mid X r=0\}$. We say that $M$ has uniform dimension $n$ (written u.dim $M=n$ ) if there exists an essential submodule $N \leq_{e} M$ that is a direct sum of $n$ uniform submodules, i.e., u.dim $M$ is the supremum of the set $\{k \mid M$ contains a direct sum of $k$ nonzero submodules\}, for more details see [21].

There are many papers on assigning graphs to groups, rings or modules, for example see $[1-4,9,18,25]$. For any $\operatorname{ring} R$ with the set of zero-divisors $Z(R)$, the zero-divisor graph of $R$, denoted by $\Gamma(R)$, is a simple graph with vertices $Z(R)^{*}=Z(R) \backslash\{0\}$ and two distinct vertices $x$ and $y$ are adjacent if and only if $x y=0$. The concept of a zerodivisor graph of a commutative ring was introduced in [13], and it was mainly concerned with coloring of rings. The above definition first appeared in the work of Anderson and Livingston [7]. This definition, unlike the earlier work of Anderson and Naseer [8] and Beck [13], dose not take zero to be a vertex of $\Gamma(R)$. The zero-divisor graph of a ring has been studied by several authors, see for example [3,4,6-8]. An ideal $I$ of a commutative ring $R$ is called an annihilating ideal if $I J=0$, for some nonzero ideal $J$ of $R$. Also the set of all annihilating ideals of $R$ is denoted by $\mathbb{A}(R)$. The notion of annihilating-ideal

\footnotetext{
*Corresponding Author.

Email addresses: beyranvand.r@lu.ac.ir (R. Beyranvand), farzi.ah@fs.lu.ac.ir (A. Farzi-Safarabadi)

Received: 16.10.2020; Accepted: 27.09.2021
} 
graph was first introduced and studied in [14]. The annihilating ideal graph of $R$, denoted by $\mathbb{A} \mathbb{G}(R)$, is a simple graph with vertices $\mathbb{A}(R)^{*}=\mathbb{A}(R) \backslash\{0\}$ and two distinct vertices $I$ and $J$ are adjacent if and only if $I J=0$. Later, it was modified and further studied by many authors, see for example $[1,2,5,23]$. Recently, the notions of zero divisor graph and annihilating-ideal graph have been extended from rings to modules in different ways. For instance, we can refer to [9] and [25]. In [9], the authors introduced and studied the annihilating-submodule graph. By the annihilating-submodule graph of $M$, denoted by $\mathbb{A} \mathbb{G}(M)$, we mean the simple graph with vertices $\{0 \neq N \leq M \mid M(N: M)(K: M)=0$, for some nonzero submodule $K$ of $M$ \} and two distinct vertices $N$ and $K$ are adjacent if and only if $M(N: M)(K: M)=0$. The authors in [10,11] and [9], investigated the basic properties of this graph and presented some related results.

In this paper, we continue to study the strongly annihilating-submodule graph of a module introduced in [15]. The strongly annihilating-submodule graph of $M$, denoted by $\mathbb{S A G}(M)$, is an undirected (simple) graph in which a nonzero submodule $N$ of $M$ is a vertex if $N(K: M)=0$ or $K(N: M)=0$, for some nonzero submodule $K \leq M$ and two distinct vertices $N$ and $K$ are adjacent if and only if $N(K: M)=0$ or $K(N: M)=0$. It is clear that if $M=R$, then $\mathbb{S A G}(R)=\mathbb{A} \mathbb{G}(R)$ and if $M$ is a multiplication $R$-module, then $\mathbb{S A G}(M)=\mathbb{A} \mathbb{G}(M)$. We compare extensively the properties of this graph with the annihilating-submodule graph.

We state some definitions and notions of graph theory used throughout this paper. Recall that for a graph $G$, the degree of a vertex $x$ in $G$ is the number of edges of $G$ incident with $x$. A graph $G$ is connected if there is a path between any two vertices of $G$. The diameter of $G$ is $\operatorname{diam}(\mathrm{G})=\sup \{d(x, y) \mid x$ and $y$ are distinct vertices of $G\}$, where $d(x, y)$ is the length of the shortest path from $x$ to $y$ in $G$ and if there is no such path, we write $d(x, y)=\infty$. The girth of a graph $G$, denoted by $\operatorname{gr}(G)$, is the smallest size of the length of cycles of $G$ and if $G$ has no cycles, we write $\operatorname{gr}(G)=\infty$. A bipartite graph $G$ is a graph whose vertices can be partitioned into two subsets $U$ and $V$ such that every edge connects a vertex in $U$ to one in $V$. Vertex sets $U$ and $V$ usually are called the parts of $G$. A complete bipartite graph is one in which every vertex in $U$ is joined to every vertex in $V$. A complete bipartite graph with parts $U$ and $V$ is called star graph if $|U|=1$ or $|V|=1$. In a graph $G$, if all the vertices of $G$ have the same degree $r$, then $G$ is called $r$-regular, or simply regular. A graph in which each pair of distinct vertices is connected by an edge is called a complete graph. A connected graph is called a tree if it has no cycles. For a graph $G$, a complete subgraph of $G$ is called a clique. The clique number, $\operatorname{cl}(G)$, is the greatest integer $n \geq 1$ such that $G$ contains a complete subgraph with $n$ vertices, and $\operatorname{cl}(G)$ is infinite if for any $n, G$ contains a complete subgraph with $n$ vertices. By $\chi(G)$, we denote the chromatic number of $G$, i.e., the minimum number of colors which can be assigned to the vertices of $G$ in such a way that every two adjacent vertices have different colors. For every graph $G$, a subset $D$ of $V(G)$ is called a dominating set if every vertex of $G$ is either in $D$ or adjacent to at least one vertex in $D$. The domination number of $G$ is the number of vertices in a smallest dominating set of $G$. A total dominating set of a graph $G$ is a subset $S$ of $V(G)$ such that every vertex is adjacent to a vertex in $S$. The total domination number of $G$ is the minimum cardinality of a total dominating set. The notations of graph theory used in the sequel can be found in [19].

The organization of this paper is as follows: In Section 2, we give more properties of $\mathbb{S A G}(M)$ and remind that $\mathbb{S A G}(M)$ can be a strict subgraph of $\mathbb{A} \mathbb{G}(M)$. It is shown that if $M$ is not a prime $R$-module, then $\mathbb{S A G}(M)$ has ACC (resp. DCC) on vertices if and only if $M$ is a Noetherian (resp. an Artinian) module (Theorem 2.8). In Section 3, we compare extensively the properties of the two graphs $\mathbb{S} \mathbb{G}(M)$ and $\mathbb{A} \mathbb{G}(M)$; in particular when $\mathbb{S A G}(M)$ ( or $\mathbb{A} \mathbb{G}(M)$ ) is a path, bipartite, tree, star, regular or complete graph. For instance, we show that $\operatorname{gr}(\mathbb{S A G}(M))=4$ if and only if $\operatorname{gr}(\mathbb{A} \mathbb{G}(M))=4$; moreover in this 
case $\mathbb{S} \mathbb{G}(M)=\mathbb{A} \mathbb{G}(M)$ (Proposition 3.3 and Theorem 3.8). Also, if $\mathbb{S} \mathbb{G}(M)$ is a tree, then either $\mathbb{S} \mathbb{G}(M)$ is a star graph or $\mathbb{S} \mathbb{A}(M)=P_{4}$; moreover, $\mathbb{S} \mathbb{G}(M)=P_{4}$ if and only if $M=F \times S$, where $F$ is a simple module and $S$ is a module with a unique nontrivial submodule (Theorem 3.10). Finally in the fourth section, we compare the (total) dominating number of $\mathbb{S} \mathbb{A}(M)$ and $\mathbb{A} \mathbb{G}(M)$ (Theorem 4.2 and Proposition 4.3). Also the dominating number and the total dominating number of $\mathbb{S A G}(M)$ are investigated (Theorem 4.4).

\section{More properties of $\mathbb{S} \mathbb{A}(M)$}

Throughout the paper, $M$ is a unitary right $R$-module and $N, K$ are nonzero submodules of $M$. In $\mathbb{S A G}(M), M$ itself can be a vertex. In fact $M$ is a vertex if and only if every nonzero submodule is a vertex, if and only if there exists a nonzero proper submodule $N$ of $M$ such that $(N: M)=\operatorname{ann}(M)$. We note that for any $R$-module $M, \mathbb{S A G}(M)$ is a subgraph of $\mathbb{A} \mathbb{G}(M)$ and if $M=R$, then the three graphs $\mathbb{S} \mathbb{G}(M), \mathbb{A} \mathbb{G}(M)$ and the annihilating-ideal graph introduced in [14] coincide. However, the following example shows that $\mathbb{S} \mathbb{G}(M)$ is a strict subgraph of $\mathbb{A} \mathbb{G}(M)$ even in the case where $M$ is semiprime (defined later) or semisimple (see part (5) in the following example). For a given $R$-module $M$, we use the notation $n(M)$ for the number of the submodules of $M$. Also the degree of a vertex $K$ in graphs $\mathbb{S} \mathbb{A} G(M)$ and $\mathbb{A} \mathbb{G}(M)$ is denoted by $\operatorname{deg}_{S}(K)$ and $\operatorname{deg}_{A}(K)$, respectively. In the following example we consider $M$ as a $\mathbb{Z}$-module.

Example 2.1. (1) Let $M=\mathbb{Z}_{2} \oplus \mathbb{Z}_{4}, N_{1}=(0) \oplus \mathbb{Z}_{4}$ and $N_{2}=(\overline{1}, \overline{1}) \mathbb{Z}$. Then $N_{1}$ and $N_{2}$ are adjacent in $\mathbb{A} \mathbb{G}(M)$, but not adjacent in $\mathbb{S} \mathbb{G}(M)$. Thus $\mathbb{S} \mathbb{G}(M)$ is different from $\mathbb{A} \mathbb{G}(M)$.

(2) Let $M=\left(\oplus_{i=1}^{n} \mathbb{Z}_{p}\right) \oplus\left(\oplus_{i=1}^{m} \mathbb{Z}_{q}\right)$ and $K=\left(\oplus_{i=1}^{n} \mathbb{Z}_{p}\right) \oplus\left(\oplus_{i=1}^{m-1} \mathbb{Z}_{q}\right)$, where $p$ and $q$ are two distinct prime numbers and $m \geq 2$. We set $N=T \oplus\left(\oplus_{i=1}^{m} \mathbb{Z}_{q}\right)$, where $T$ is a nonzero proper submodule of $\oplus_{i=1}^{n} \mathbb{Z}_{p}$. Then $M(N: M)(K: M)=M(p \mathbb{Z})(q \mathbb{Z})=0$. However, $N(K: M)=N(q \mathbb{Z}) \neq 0$ and $K(N: M)=K(p \mathbb{Z}) \neq 0$. Thus $N$ and $K$ are adjacent in $\mathbb{A} \mathbb{G}(M)$, but not adjacent in $\mathbb{S A G}(M)$ and hence $\operatorname{deg}_{A}(K)-\operatorname{deg}_{S}(K) \geq n\left(\oplus_{i=1}^{n} \mathbb{Z}_{p}\right)-2$.

(3) Let $M=\mathbb{Z}_{p} \oplus \mathbb{Z}_{p} \oplus\left(\oplus_{i=1}^{m} \mathbb{Z}_{q}\right)$ and $K=\mathbb{Z}_{p} \oplus \mathbb{Z}_{p} \oplus\left(\oplus_{i=1}^{m-1} \mathbb{Z}_{q}\right)$, where $p$ and $q$ are two distinct prime numbers and $m \geq 2$. Since by [26, Corollary 4.4], $n\left(\mathbb{Z}_{p} \oplus \mathbb{Z}_{p}\right)=p+3$, the part (2) implies that $\operatorname{deg}_{A}(K)-\operatorname{deg}_{S}(K) \geq p+1$.

(4) In $\mathbb{Z}_{16}, \mathbb{S} \mathbb{A}\left(\mathbb{Z}_{16}\right)=\mathbb{A} \mathbb{G}\left(\mathbb{Z}_{16}\right)$ is the star graph $N_{1}-N_{3}-N_{2}$, where $N_{1}=2 \mathbb{Z}_{16}$, $N_{2}=4 \mathbb{Z}_{16}$ and $N_{3}=8 \mathbb{Z}_{16}$.

(5) Let $M=\left(\oplus_{i=1}^{2} \mathbb{Z}_{2}\right) \oplus\left(\oplus_{i=1}^{2} \mathbb{Z}_{3}\right), N=\mathbb{Z}_{2} \oplus\left(\oplus_{i=1}^{2} \mathbb{Z}_{3}\right)$ and $K=\left(\oplus_{i=1}^{2} \mathbb{Z}_{2}\right) \oplus \mathbb{Z}_{3}$. Clearly $N$ and $K$ are adjacent in $\mathbb{A} \mathbb{G}(M)$, but not adjacent in $\mathbb{S} \mathbb{G}(M)$.

An $R$-module $M$ is called prime if $\operatorname{ann}_{R}(M)=\operatorname{ann}_{R}(N)$, for any nonzero submodule $N$ of $M$. Also $M$ is called weakly prime (resp. semiprime), if $\operatorname{ann}_{R}(N)$ is a prime (resp. semiprime) ideal of $R$, for any nonzero submodule $N$ of $M$. The dual of notions prime and weakly prime for modules are second and weakly second, respectively. Indeed $M$ is called second if $\operatorname{ann}_{R}(M)=\operatorname{ann}_{R}(M / N)$, for any proper submodule $N$ of $M$. Also $M$ is called weakly second, if $\operatorname{ann}_{R}(M / N)$ is a prime ideal of $R$, for any proper submodule $N$ of $M$. Clearly, any prime module is weakly prime and also any second module is weakly second. For more details about these notions, the reader is referred to $[12,16,17]$.

Example 2.2. (1) If $M=\oplus_{i=1}^{n} S_{i}$, where $S_{i}$ 's are isomorphic simple $R$-modules, then $\mathbb{S A G}(M)$ is a complete graph such that every nonzero submodule of $M$ is a vertex and so $\mathbb{S A G}(M)=\mathbb{A} \mathbb{G}(M)$.

(2) It is easy to see that $\mathbb{S A G}(M)$ is the empty graph if and only if $M$ is a prime module and not vertex. 
Proposition 2.3. If one of the following conditions holds, then the two graphs $\mathbb{S A G}(M)$ and $\mathbb{A} \mathbb{G}(M)$ coincide.

(1) $M$ is prime.

(2) $M$ is weakly prime.

(3) $M$ is second.

(4) $M$ is weakly second.

(5) $M$ is a multiplication $R$-module (i.e., for every submodule $N$ of $M$ there exists an ideal $I$ of $R$ such that $N=M I$ ).

(6) $M$ is a cyclic R-module.

Proof. Clear.

The following useful lemmas will be used frequently in this paper.

Lemma 2.4. [15, Lemma 2.1] (1) If $N$ and $K$ are adjacent in $\mathbb{S A G}(M)$, then $N_{1}$ and $K_{1}$ are also adjacent in $\mathbb{S} \mathbb{A}(M)$ for every $0 \neq N_{1} \leq N$ and $0 \neq K_{1} \leq K$ with $N_{1} \neq K_{1}$.

(2) If $N \cap K=0$, then $N$ and $K$ are adjacent in $\mathbb{S A G}(M)$.

(3) If $N$ is not a vertex in $\mathbb{S A G}(M)$, then $N \leq_{e} M$.

The converse of (3) in the above lemma is not true. Indeed, if we consider $\mathbb{Z}_{12}$ as a $\mathbb{Z}$-module, then $2 \mathbb{Z}_{12} \leq_{e} \mathbb{Z}_{12}$. However, $2 \mathbb{Z}_{12}$ and $6 \mathbb{Z}_{12}$ are adjacent and so $2 \mathbb{Z}_{12}$ is a vertex in $\mathbb{S A G}\left(\mathbb{Z}_{12}\right)$.

The following lemma shows that $V(\mathbb{S A} \mathbb{G}(M))=V(\mathbb{A} \mathbb{G}(M))$.

Lemma 2.5. [15, Lemma 2.2] If $N$ and $K$ are adjacent in $\mathbb{A} \mathbb{G}(M)$, then the following statements hold.

(1) $N$ and $K$ are adjacent in $\mathbb{S A G}(M)$ or there exists a nonzero submodule of $N \cap K$ such that is adjacent to both $N$ and $K$ in $\mathbb{S A G}(M)$.

(2) There exists a nonzero submodule of $N$ that is adjacent to $K$ in $\mathbb{S A G}(M)$.

Corollary 2.6. Let $N$ and $K$ be adjacent in $\mathbb{A} \mathbb{G}(M)$ and $N$ be a minimal submodule of $M$. Then $N$ and $K$ are adjacent in $\mathbb{S A G}(M)$.

Proposition 2.7. Let $M$ be an $R$-module and $I$ be an ideal of $R$.

(1) If $V(\mathbb{S A G}(M)) \neq \emptyset$, then every minimal submodule of $M$ is a vertex.

(2) If $M I-N$ is an edge in $\mathbb{A} G(M)$, then $M I-N$ is an edge in $\mathbb{S A G}(M)$.

(3) If $\mathbb{A} \mathbb{G}(M)$ is a triangle-free graph or contains no cycle, then $\mathbb{S} \mathbb{G}(M)=\mathbb{A} \mathbb{G}(M)$.

Proof. (1). Let $N$ be a minimal submodule of $M$. Then for every nonzero submodule $K$ of $M, N \cap K=0$ or $N \subseteq K$. If $N \cap K=0$, then $N$ and $K$ are adjacent, and we are done. Thus we may assume that $N \subseteq K$, for any nonzero submodule $K$ of $M$. Now let $K \in V(\mathbb{S A G}(M))$. Then there exists $0 \neq K^{\prime} \leq M$ such that $K\left(K^{\prime}: M\right)=0$ or $K^{\prime}(K: M)=0$. Thus $N\left(K^{\prime}: M\right)=0$ or $K^{\prime}(N: M)=0$, as desired.

(2). Since $M I-N$ is an edge in $\mathbb{A} \mathbb{G}(M)$, we have $M(M I: M)(N: M)=0$. Clearly $M(M I: M)=M I$. Thus $M I(N: M)=0$, as desired.

(3). It is clear by Lemma 2.5 .

Theorem 2.8. If $M$ is not a prime $R$-module, then $\mathbb{S A G}(M)$ has ACC (resp. DCC) on vertices if and only if $M$ is a Noetherian (resp. an Artinian) module.

Proof. Suppose that $\mathbb{S A G}(M)$ has ACC (resp. DCC) on vertices. Since $M$ is not a prime module, there exists $r \in R$ and $m \in M$ such that $m r=0$ but $m \neq 0$ and $r \notin \operatorname{ann}(M)$. Since $M r\left(\operatorname{ann}_{M}(r): M\right) \subseteq \operatorname{ann}_{M}(r) r=0$, every nonzero submodule in $\operatorname{ann}_{M}(r)$ and in $M r$ is a vertex. This implies that the $R$-modules $\operatorname{ann}_{M}(r)$ and $M r$ have ACC (resp. DCC) on submodules. Now $M r \cong M / \operatorname{ann}_{M}(r)$ implies that $M$ is a Noetherian (resp. an Artinian) module. The converse is clear. 
Corollary 2.9. [15, Theorem 2.4] For any $R$-module $M, \mathbb{S A} \mathbb{G}(M)$ is a connected graph with $\operatorname{diam}(\mathbb{S A G}(M)) \leq 3$.

Theorem 2.10. [15, Theorem 2.5] For any $R$-module $M$, if $\mathbb{S A G}(M)$ contains a cycle, then $\operatorname{gr}(\mathbb{S A G}(M)) \leq 4$.

\section{Comparison of $\mathbb{S} \mathbb{G}(M)$ and $\mathbb{A} \mathbb{G}(M)$}

At the beginning of this section, we compare the girth of two graphs $\mathbb{A} \mathbb{G}(M)$ and $\mathbb{S A G}(M)$.

Proposition 3.1. Let $M$ be an R-module with $\mathrm{u} \cdot \operatorname{dim}(M) \geq 2$. Then

$$
\operatorname{gr}(\mathbb{S} \mathbb{G}(M))=3 \Longleftrightarrow \operatorname{gr}(\mathbb{A} \mathbb{G}(M))=3 .
$$

Proof. Let $\operatorname{gr}(\mathbb{A} G(M))=3$ and $N_{1}-N_{2}-N_{3}-N_{1}$ be a cycle in $\mathbb{A} \mathbb{G}(M)$. If this cycle is also a cycle in $\mathbb{S A G}(M)$, there is nothing to prove. Thus without loss of generality, we may assume that $N_{1}$ and $N_{2}$ are not adjacent in $\mathbb{S A} \mathbb{G}(M)$. By Lemma 2.5, there exists $L \leq N_{1} \cap N_{2}$ such that $N_{1}-L-N_{2}$ is a path in $\mathbb{S A G}(M)$. If there exists $0 \neq L_{1} \lesseqgtr L$, then $L-L_{1}-N_{1}-L$ is a cycle in $\mathbb{S A G}(M)$. Now assume that $L$ is minimal and $L_{2} \leq M$ is a complement of $L$ in $M$. Then $L \oplus L_{2} \leq_{e} M$ and since $M$ is not uniform, $L_{2} \neq 0$. If $N_{1} \cap L_{2}=0$, then $L-L_{2}-N_{1}-L$ is a cycle in $\mathbb{S} \mathbb{G}(M)$. Thus we assume that $N_{1} \cap L_{2} \neq 0$ and consider the following cases.

Case 1: $N_{1} \cap L_{2} \neq L_{2}$. Then since $N_{2}-N_{1} \cap L_{2}$ is an edge in $\mathbb{A} \mathbb{G}(M)$, by Lemma 2.5 $L-N_{2}-N_{1} \cap L_{2}-L$ is a cycle in $\mathbb{S A G}(M)$ or there exists $K \leq N_{2} \cap\left(N_{1} \cap L_{2}\right)$ such that $N_{2}-K-N_{1} \cap L_{2}$ is a path in $\mathbb{S A G}(M)$. In the latter, $L-N_{2}-K-L$ is a cycle in $\mathbb{S A G}(M)$.

Case 2: $N_{1} \cap L_{2}=L_{2}$. Then $L_{2} \subsetneq N_{1}$ and since $N_{2}-L_{2}$ is an edge in $\mathbb{A} \mathbb{G}(M)$, by Lemma $2.5 L-N_{2}-L_{2}-L$ in $\mathbb{S A G}(M)$ or there exists $K \leq N_{2} \cap L_{2}$ such that $N_{2}-K-L_{2}$ is a path in $\mathbb{S A G}(M)$. In the latter, $L-N_{2}-K-L$ is a cycle in $\mathbb{S A G}(M)$. The converse is clear.

We have not found any example of a module that $\operatorname{gr}(\mathbb{A} \mathbb{G}(M))=3$ and $\operatorname{gr}(\mathbb{S} \mathbb{G}(M)) \neq$ 3. The lack of such counterexample together with the above proposition motivates the following fundamental conjecture.

Conjecture 3.2. For any $R$-module $M, \operatorname{gr}(\mathbb{S} \mathbb{G}(M))=3$ if and only if $\operatorname{gr}(\mathbb{A} \mathbb{G}(M))=3$.

Proposition 3.3. For any $R$-module $M, \operatorname{gr}(\mathbb{S A} \mathbb{G}(M))=4$ if and only if $\operatorname{gr}(\mathbb{A} \mathbb{G}(M))=4$.

Proof. Let $\operatorname{gr}(\mathbb{A G}(M))=4$ and $N_{1}-N_{2}-N_{3}-N_{4}-N_{1}$ be a cycle in $\mathbb{A} \mathbb{G}(M)$. We claim that $N_{1} \cap N_{2}=N_{2} \cap N_{3}=N_{3} \cap N_{4}=N_{4} \cap N_{1}=0$ and this implies that $N_{1}-N_{2}-N_{3}-$ $N_{4}-N_{1}$ is also a cycle in $\mathbb{S} \mathbb{A} G(M)$. On the contrary, assume that $N_{1} \cap N_{2} \neq 0$. Then the following cases can occur.

Case 1: $N_{1} \cap N_{2} \notin\left\{N_{1}, N_{2}\right\}$. Then $N_{1}-N_{1} \cap N_{2}-N_{2}-N_{1}$ is a cycle in $\mathbb{A} \mathbb{G}(M)$, a contradiction.

Case 2: $N_{1} \cap N_{2}=N_{1}$. Then $N_{1} \subseteq N_{2}$ and so $N_{1}-N_{2}-N_{3}-N_{1}$ is a cycle in $\mathbb{A} \mathbb{G}(M)$, a contradiction.

Case 3: $N_{1} \cap N_{2}=N_{2}$. Then $N_{2} \subseteq N_{1}$ and so $N_{1}-N_{2}-N_{4}-N_{1}$ is a cycle in $\mathbb{A} \mathbb{G}(M)$, a contradiction. Thus the claim is proved and so $\operatorname{gr}(\mathbb{S A G}(M))=4$.

Conversely, let $\operatorname{gr}(\mathbb{S A G}(M))=4$ and $N_{1}-N_{2}-N_{3}-N_{4}-N_{1}$ be a cycle in $\mathbb{S A G}(M)$. By [9, Theorem 3.4], $\operatorname{gr}(\mathbb{A} \mathbb{G}(M)) \leq 4$. First, we claim that $N_{1}$ and $N_{3}$ are not adjacent in $\mathbb{A} G(M)$. On the contrary, assume that $N_{1}$ and $N_{3}$ adjacent in $\mathbb{A} \mathbb{G}(M)$. By Lemma 2.5, there exists $L \leq N_{1} \cap N_{3}$ such that $L$ is adjacent both $N_{1}$ and $N_{3}$ in $\mathbb{S A G}(M)$. If $L \notin\left\{N_{2}, N_{4}\right\}$, then $N_{3}-L-N_{4}-N_{3}$ is a cycle in $\mathbb{S A G}(M)$, a contradiction. If $L=N_{2}$ or $L=N_{4}$, then $N_{1}-N_{2}-N_{4}-N_{1}$ is a cycle in $\mathbb{S A G}(M)$, a contradiction. Similarly, 
we can show that $N_{2}$ and $N_{4}$ are not adjacent in $\mathbb{A} \mathbb{G}(M)$. Now if $\operatorname{gr}(\mathbb{A} \mathbb{G}(M))=3$, then by Proposition 3.1, $M$ must be uniform and as in proof of that proposition, we will have a triangle $N_{5}-N_{6}-N_{7}-N_{5}$ in $\mathbb{A} \mathbb{G}(M)$ such that $N_{5}$ is a minimal essential submodule of $M$ and so for every vertex $K, N_{5} \subseteq K$. If $N_{5} \neq N_{i}$ for $i \in\{1,2,3,4\}$, then $N_{5}-N_{1}-N_{2}-N_{5}$ is a cycle in $\mathbb{S A G}(M)$, because $N_{5} \subseteq N_{4}$ and also $N_{5} \subseteq N_{3}$, a contradiction. If $N_{5}=N_{i}$ for some $i \in\{1,2,3,4\}$, without loss of generality, we may assume that $N_{5}=N_{2}$. Then $N_{1}-N_{2}-N_{4}-N_{1}$ is a cycle in $\mathbb{S} \mathbb{G}(M)$, because $N_{2} \subseteq N_{1}$, which again is a contradiction.

Corollary 3.4. Let $M$ be an $R$-module with u.dim $(M) \geq 2$. Then $\mathbb{A} \mathbb{G}(M)$ is a tree if and only if $\mathbb{S A G}(M)$ is a tree.

Proof. Let $\mathbb{S} \mathbb{G}(M)$ be a tree. If $\mathbb{A} \mathbb{G}(M)$ contains a cycle, then by [9, Theorem 3.4], $\operatorname{gr}(\mathbb{A} G(M)) \leq 4$. Now by Proposition 3.1 and Proposition 3.3, we have $\operatorname{gr}(\mathbb{S} \mathbb{G}(M))=4$ or $\operatorname{gr}(\mathbb{S} \mathbb{G}(M))=3$, a contradiction. Thus $\mathbb{A} \mathbb{G}(M)$ is also tree. Clearly, if $\mathbb{A} \mathbb{G}(M)$ is tree, then so is $\mathbb{S A} \mathbb{G}(M)$.

Proposition 3.5. (1) If $\mathbb{A} \mathbb{G}(M)$ is a bipartite graph, then so is $\mathbb{S} \mathbb{G}(M)$.

(2) If $\mathbb{S A G}(M)$ is a bipartite graph with parts $V_{1}$ and $V_{2}$ such that $\left|V_{1}\right| \geq 2$ and $\left|V_{2}\right| \geq 2$, then $\mathbb{A} \mathbb{G}(M)$ is also bipartite.

(3) If $\mathbb{S A G}(M)$ is a bipartite graph with u.dim $(M) \geq 2$, then $\mathbb{A} \mathbb{G}(M)$ is also bipartite.

Proof. (1) is clear.

(2). Let $\mathbb{S A G}(M)$ be a bipartite graph with the conditions said in (2). Suppose, on the contrary, there exist $N$ and $K$ in $V_{1}$ such that $N-K$ is an edge in $\mathbb{A} \mathbb{G}(M)$. By Lemma 2.5, there exists $L \leq N \cap K$ such that both $N$ and $K$ are adjacent to $L$ in $\mathbb{S A G}(M)$. Clearly $L \in V_{2}$. By hypothesis, we can choose $L \neq T \in V_{2}$ and consider $d_{S}(T, K)$ and $d_{S}(T, N)$, where $d_{S}(T, N)$ is the length of the shortest path from $T$ to $N$ in $\mathbb{S A G}(M)$. If $d_{S}(T, N)=1$, then $L \subseteq N \cap K$ implies that $T-L$ is an adge in $\mathbb{S A} \mathbb{G}(M)$, a contradiction. Similarly, $d_{S}(T, K) \neq 1$. Since $\mathbb{S A G}(M)$ is connected with diameter at most 3 and it is bipartite, we conclude that $d_{S}(T, N)=d_{S}(T, K)=3$. Thus $T-T_{1}-P-K$ is a path in $\mathbb{S A G}(M)$, for some $T_{1} \in V_{1}$ and $P \in V_{2}$. If $P \neq L$, then $P$ is adjacent to $L$ in $\mathbb{S A} \mathbb{G}(M)$, a contradiction, since $P$ is adjacent to $K$ in $\mathbb{S A G}(M)$. Thus $P=L$. Clearly $N \cap T_{1} \neq 0$ and $L$ is adjacent to $N \cap T_{1}$ in $\mathbb{S A G}(M)$, so $N \cap T_{1} \in V_{1}$. Now one of the following cases may occur.

Case 1: $N \cap T_{1}=N$. Then $N \subseteq T_{1}$ and since $T$ is adjacent to $T_{1}$ in $\mathbb{S A G}(M)$, so $T$ is adjacent to $N$ in $\mathbb{S} \mathbb{G}(M)$, a contradiction.

Case 2: $N \cap T_{1}=K$. Then $K \subseteq T_{1}$ and since $T$ is adjacent to $T_{1}$ in $\mathbb{S A G}(M)$, so $T$ is adjacent to $K$ in $\mathbb{S A G}(M)$, a contradiction.

Case 3: $N \cap T_{1}=T_{1}$. Then $T_{1} \subseteq N$ and since $K$ is adjacent to $N$ in $\mathbb{A} \mathbb{G}(M)$, we conclude that $K$ is adjacent to $T_{1}$ in $\mathbb{A} \mathbb{G}(M)$. Now by Lemma 2.5, there exists $T_{2} \lesseqgtr T_{1}$ such that $K$ is adjacent to $T_{2}$ in $\mathbb{S A G}(M)$. Thus $T_{2} \in V_{2}$ and it is easy to see that $T_{2}=L$. This means $L \subseteq T_{1}$ and hence $T$ is adjacent to $L$ in $\mathbb{S A G}(M)$, because $T$ is adjacent to $T_{1}$ in $\mathbb{S} \mathbb{A}(M)$, a contradiction.

Case 4: $N \cap T_{1} \notin\left\{N, K, T_{1}\right\}$. By replacing $T_{1}$ with $N \cap T_{1}$ in Case 3 , we get a contradiction. Thus $\mathbb{A} \mathbb{G}(M)$ is a bipartite graph, as desired.

(3). If $\mathbb{A} \mathbb{G}(M)$ is not bipartite, then by Lemma 2.5 , it contains a triangle and $\operatorname{sog} \operatorname{gr}(\mathbb{A} \mathbb{G}(M))$ $=3$. Now by Proposition 3.1, $\operatorname{gr}(\mathbb{S A G}(M))=3$, a contradiction.

Corollary 3.6. Suppose that one of the two graphs $\mathbb{A} \mathbb{G}(M)$ and $\mathbb{S} \mathbb{G}(M)$ is bipartite with parts $V_{1}$ and $V_{2}$. If one of the following holds, then $\mathbb{A} \mathbb{G}(M)$ and $\mathbb{S} \mathbb{G}(M)$ coincide.

(1) $\left|V_{1}\right| \geq 2$ and $\left|V_{2}\right| \geq 2$.

(2) u.dim $(M) \geq 2$.

Proof. Follows from Proposition 2.7(3) and Proposition 3.5 parts (2) and (3). 
A similar result of the following has appeared in [24, Lemma 3.3]. Here, we give a shorter proof of this fact.

Lemma 3.7. If $\mathbb{S A G}(M)$ contains a cycle of odd length, then it contains a triangle.

Proof. Using induction, we show that for every cycle of odd length $2 n+1 \geq 5$, there exists a cycle with length $2 k+1$ such that $k<n$. Assume that $N_{1}-N_{2}-\cdots-N_{2 n+1}-N_{1}$ is a cycle with odd length $2 n+1$. If two distinct non consecutive $N_{i}$ and $N_{j}$ are adjacent, the proof is complete. Otherwise, we set $0 \neq L=N_{1} \cap N_{3}$. Then by Lemma $2.4, L \neq N_{i}$ for all $1 \leq i \leq 2 n+1$ and $L$ is adjacent to both $N_{4}$ and $N_{2 n+1}$. Hence we have the cycle $N_{2 n+1}-L-N_{4}-N_{5}-\cdots-N_{2 n+1}$, which is the desired cycle.

Theorem 3.8. If $\operatorname{gr}(\mathbb{S} \mathbb{G}(M))=4$ or $\operatorname{gr}(\mathbb{A} G(M))=4$, then $\mathbb{A} \mathbb{G}(M)$ and $\mathbb{S} \mathbb{G}(M)$ coincide.

Proof. Follows from Propositions 3.3 and 2.7(3).

For any $R$-module $M$, since $\operatorname{diam}(\mathbb{A} G(M)) \leq 3$ and $\operatorname{diam}(\mathbb{S A G}(M)) \leq 3$, if either $\mathbb{A} \mathbb{G}(M)=P_{n}$ or $\mathbb{S A G}(M)=P_{n}$, then $n \leq 4$. Now we have the following interesting proposition.

Proposition 3.9. Let $M$ be an R-module. Then

(1) For $n \neq 3, \mathbb{A} \mathbb{G}(M)=P_{n}$ if and only if $\mathbb{S} \mathbb{G}(M)=P_{n}$.

(2) If $M$ is not uniform, then $\mathbb{A} \mathbb{G}(M)=P_{3}$ if and only if $\mathbb{S A} \mathbb{G}(M)=P_{3}$

Proof. (1). For $n=1$ and $n=2$, the proof is clear. Let $\mathbb{S} \mathbb{G}(M)$ be a path, say, $N_{1}-N_{2}-N_{3}-N_{4}$. If $\mathbb{A} \mathbb{G}(M) \neq \mathbb{S} \mathbb{G}(M)$, since the set of all vertices of $\mathbb{A} \mathbb{G}(M)$ is equal to the set of all vertices of $\mathbb{S} \mathbb{G}(M), \mathbb{A} \mathbb{G}(M)$ must contain a cycle. Thus by $[9$, Theorem 3.4], $\operatorname{gr}(\mathbb{A} \mathbb{G}(M)) \leq 4$. If $\operatorname{gr}(\mathbb{A} \mathbb{G}(M))=4$, then by Proposition 3.3, $\operatorname{gr}(\mathbb{S} \mathbb{G}(M))=4$, a contradiction. If $\operatorname{gr}(\mathbb{A} \mathbb{G}(M))=3$, then without loss of generality, we may assume $N_{1}$ and $N_{3}$ are adjacent in $\mathbb{A} \mathbb{G}(M)$. Then by Lemma 2.5, there exists $L \subseteq N_{3}$ such that $N_{1}-L$ is an edge in $\mathbb{S A G}(M)$. Since $\mathbb{S A G}(M)=P_{4}$, we will have $L=N_{2}$ and so $N_{2} \subseteq N_{3}$. Now $N_{4}$ and $N_{2}$ must be adjacent in $\mathbb{S A G}(M)$, a contradiction. The converse is clear.

(2). Let $\mathbb{S A G}(M)$ be a path, say, $N_{1}-N_{2}-N_{3}$ and $\mathbb{A} \mathbb{G}(M) \neq \mathbb{S A} \mathbb{G}(M)$. Then $\mathbb{A} \mathbb{G}(M)$ must be a triangle. Thus $\operatorname{gr}(\mathbb{A} \mathbb{G}(M))=3$ and so by Proposition 3.1, $\operatorname{gr}(\mathbb{S} \mathbb{G}(M))=3$, a contradiction. The converse is clear.

Theorem 3.10. If $\mathbb{S A G}(M)$ is a tree, then either $\mathbb{S A G}(M)$ is a star graph or $\mathbb{S} \mathbb{G}(M)=$ $P_{4}$. Moreover, $\mathbb{S A G}(M)=P_{4}$ if and only if $M=F \times S$, where $F$ is a simple module and $S$ is a module with a unique non-trivial submodule.

Proof. If $M$ is a vertex of $\mathbb{S A G}(M)$, then there exists nonzero submodule $N \leq M$ such that $M(N: M)=0$ and so $K(N: M)=0$ for every nonzero submodule $K \leq M$. Thus every vertex is adjacent to $N$ and since $\mathbb{S A G}(M)$ is tree, it must be a star graph. Now we assume that $M$ is not a vertex of $\mathbb{S A G}(M)$ and $\mathbb{S A G}(M)$ is not star. Now by [19, Proposition 1.6.1], $\mathbb{S} \mathbb{G}(M)$ is a bipartite graph with parts $V_{1}$ and $V_{2}$ such that $\left|V_{1}\right| \geq 2$ and $\left|V_{2}\right| \geq 2$. By Proposition 3.5, $\mathbb{A} \mathbb{G}(M)$ is a bipartite graph and so by Corollary 3.6, $\mathbb{A} \mathbb{G}(M)$ and $\mathbb{A} \mathbb{G}(M)$ coincide. Now by [10, Theorem 2.7], $\mathbb{A} G(M)=P_{4}$ and hence $\mathbb{S A G}(M)=P_{4}$. For the latter assertion, if $\mathbb{S A} \mathbb{G}(M)=P_{4}$, then by Proposition 3.9, $\mathbb{A} G(M)=P_{4}$ and so by [10, Theorem 2.7], the proof is complete.

Theorem 3.11. Let $R$ be an Artinian ring and $\mathbb{S A G}(M)$ be a bipartite graph. Then $\mathbb{S A G}(M)$ is a star graph or $\mathbb{S} \mathbb{G}(M)=P_{4}$. Moreover, if $R$ is an Artinian local ring, then $\mathbb{S A G}(M)$ is a star graph.

Proof. Suppose that $\mathbb{S} \mathbb{G}(M)$ is not a star graph, then $\mathbb{S A} \mathbb{G}(M)$ is a bipartite graph with parts $V_{1}$ and $V_{2}$ such that $\left|V_{1}\right| \geq 2$ and $\left|V_{2}\right| \geq 2$. By Corollary 3.6, $\mathbb{S} \mathbb{G}(M)=\mathbb{A} \mathbb{G}(M)$. Now by [10, Theorem 2.8], $\mathbb{S A G}(M)=P_{4}$. Let $m$ be a unique maximal ideal of $R$. Since $R$ 
is an Artinian ring, there exists a natural number $k$ such that $M m^{k}=0$ and $M m^{k-1} \neq 0$. Clearly $M m^{k-1}(N: M)=0$ for each submodule $N$ of $M$ and so $M m^{k-1}$ is adjacent to every other vertex of $\mathbb{S} \mathbb{G}(M)$. Thus $\mathbb{S} \mathbb{G}(M)$ is a star graph.

Proposition 3.12. Let $M=M_{1} \times M_{2}$ where $M_{1}=M e \neq 0, M_{2}=M(1-e) \neq 0$ and e be an idempotent element of $R$. If $\mathbb{S A G}(M)$ is a triangle-free graph, then one of the following statements holds.

(1) Both $M_{1}$ and $M_{2}$ are prime $R$-modules.

(2) One $M_{i}$ is a prime module for $i=1,2$ and the other one is a module with unique non-trivial submodule. Moreover, $\mathbb{S} \mathbb{G}(M)$ has no cycle if and only if $M=F \times S$ or $M=F \times D$, where $F$ is a simple module, $S$ is a module with a unique non-trivial submodule and $D$ is a prime module.

Proof. Clearly $M$ is not a uniform module. If $\mathbb{S A G}(M)$ is a triangle-free graph, then by Proposition 3.1, $\mathbb{A} \mathbb{G}(M)$ is a triangle-free graph. Thus by [10, Theorem 2.6], (1) or (2) holds. Now suppose that $\mathbb{S A G}(M)$ has no cycle. Then by Theorem $3.10, \mathbb{S A} \mathbb{G}(M)$ is a star graph or $\mathbb{S A G}(M)=P_{4}$ and also $\mathbb{S A G}(M)=P_{4}$ if and only if $M=F \times S$, where $F$ is a simple module and $S$ is a module with a unique non-trivial submodule. If $\mathbb{S A G}(M)$ is a star graph, then by Proposition 3.1, $\mathbb{A} \mathbb{G}(M)$ is a star graph and so $\mathbb{S} \mathbb{G}(M)=\mathbb{A} \mathbb{G}(M)$. Now by [10, Theorem 2.6], we are done. The converse is trivial.

Lemma 3.13. If $\mathbb{S} \mathbb{G}(M)$ is a regular graph of degree $r$, then so is $\mathbb{A} \mathbb{G}(M)$; in particular $\mathbb{A} \mathbb{G}(M)=\mathbb{S A} \mathbb{G}(M)$.

Proof. Let $\mathbb{S A G}(M)$ be a regular graph of degree $r$. If $\mathbb{S A G}(M)$ is a complete graph, then it is clear that $\mathbb{A} \mathbb{G}(M)$ is also complete. Now assume that $\mathbb{S A G}(M)$ is not complete. Then we show that $\mathbb{A} \mathbb{G}(M)=\mathbb{S} \mathbb{G}(M)$. Suppose, on the contrary, there exist two vertices $N$ and $K$ that are adjacent in $\mathbb{A} \mathbb{G}(M)$ but are not adjacent in $\mathbb{S} \mathbb{G}(M)$. Then by Lemma 2.5 , there exists $L \leq N \cap K$ such that both $N$ and $K$ are adjacent to $L$ in $\mathbb{S A G}(M)$. On the other hand since $L \subseteq N$, every vertex that is adjacent to $N$, is also adjacent to $L$. Thus we conclude that $\operatorname{deg}_{S}(L) \geq r-1+2=r+1$, a contradiction.

Theorem 3.14. Let $\operatorname{ann}(M)$ be a nil ideal of $R$. If $\mathbb{S A G}(M)$ is a regular graph of finite degree, then $\mathbb{S} \mathbb{A}(M)$ is a complete graph; in particular $\mathbb{A} \mathbb{G}(M)=\mathbb{S} \mathbb{G}(M)$.

Proof. Suppose that $\mathbb{S} \mathbb{A}(M)$ is a regular graph of degree $r$. Then by Lemma 3.13, $\mathbb{A} \mathbb{G}(M)$ is also a regular graph of degree $r$ and $\mathbb{A} \mathbb{G}(M)=\mathbb{S} \mathbb{A}(M)$. Now by $[10$, Theorem 2.9], $\mathbb{A} \mathbb{G}(M)$ is a complete graph and so is $\mathbb{S} \mathbb{A}(M)$.

In the following theorem for any vertex $K$ in the graph $\mathbb{A} \mathbb{G}(M)$, we denote by $N_{A}(K)$, the set of all vertices of $G$ adjacent to $K$.

Theorem 3.15. Let $\mathbb{A} \mathbb{G}(M)$ be a regular graph of degree $r$. If $|V(\mathbb{A} \mathbb{G}(M))| \geq r+2$, then $\mathbb{S A G}(M)$ is also regular; in particular $\mathbb{S} \mathbb{G}(M)=\mathbb{A} \mathbb{G}(M)$.

Proof. Clearly $r \neq 1$. Suppose that $N$ and $K$ are adjacent in $\mathbb{A} \mathbb{G}(M)$. We claim that $N \cap K=0$ and so $N$ and $K$ are adjacent in $\mathbb{S A G}(M)$. Suppose, on the contrary, $N \cap K \neq 0$. One of the following cases holds.

Case 1: $N \cap K \notin\{N, K\}$. Then we may assume $N_{A}(K)=\left\{N, N \cap K, K_{1}, K_{2}, \cdots, K_{r-2}\right\}$. As $N_{A}(K) \backslash\{N \cap K\} \subseteq N_{A}(N \cap K)$ and $\mathbb{S A G}(M)$ is a regular graph of degree $r$, we have $N_{A}(N \cap K)=\left\{N, K, K_{1}, K_{2}, \cdots, K_{r-2}\right\}$. This implies that $N_{A}(N)=\{K, N \cap$ $\left.K, K_{1}, K_{2}, \cdots, K_{r-2}\right\}$. Now, since $|V(\mathbb{A} \mathbb{G}(M))| \geq r+2$, we consider a vertex $L$ such that $L \notin\left\{N, K, N \cap K, K_{1}, K_{2}, \cdots, K_{r-2}\right\}$. Clearly $L$ is not adjacent to any of the vertices $N, K$ and $N \cap K$. Thus there exists a subset $\left\{L_{1}, L_{2}\right\} \subseteq V(\mathbb{A} \mathbb{G}(M)) \backslash\{N, K, N \cap$ $\left.K, K_{1}, K_{2}, \cdots, K_{r-2}\right\}$ such that $L_{i}$ is adjacent to $L$ in $\mathbb{A G}(M)$, for $1 \leq i \leq 2$. It is easy to check that $0 \neq N \cap L \notin\left\{L, N, K, N \cap K, K_{1}, K_{2}, \cdots, K_{r-2}\right\}$. Now since $K$ is adjacent to

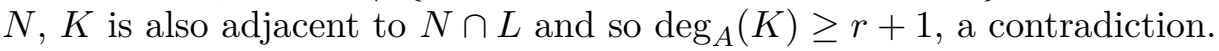


Case 2: $N \cap K=N$. Then $N \subseteq K$. Suppose $N_{A}(K)=\left\{N, K_{1}, K_{2}, \cdots, K_{r-1}\right\}$. Then $N_{A}(N)=\left\{K, K_{1}, K_{2}, \cdots, K_{r-1}\right\}$. Now since $|V(\mathbb{A} \mathbb{G}(M))| \geq r+2$, there exists a vertex $L$ such that $L \notin\left\{N, K, K_{1}, K_{2}, \cdots, K_{r-1}\right\}$. Clearly $L$ is not adjacent to any of the vertices $N$ and $K$. Thus there exists a vertex $L_{1}$ such that is adjacent to $L$ in $\mathbb{A} \mathbb{G}(M)$ and $L_{1} \notin\left\{N, K, K_{1}, K_{2}, \cdots, K_{r-1}\right\}$. It is easy to check that $0 \neq N \cap L \notin\left\{L, N, K, K_{1}, K_{2}, \cdots, K_{r-1}\right\}$. Clearly $N \cap L$ is adjacent to $K$ and so $\operatorname{deg}_{A}(K) \geq r+1$, a contradiction.

Case 3: $N \cap K=K$. It is similar to Case 2 .

For any $N \leq M$, the prime radical $\operatorname{rad}_{M}(N)$ or simply $\operatorname{rad}(N)$ is defined to be the intersection of all prime submodules of $M$ containing $N$, and in case $N$ is not contained in any prime submodule, $\operatorname{rad}_{M}(N)$ is defined to be $M$. Also the set of all minimal prime submodules of $M$ is denoted by $\operatorname{Min}(M)$.

Proposition 3.16. Let $M$ be a finitely generated module, $\operatorname{ann}(M)$ be a nil ideal and $|\operatorname{Min}(M)|=1$. If $\mathbb{S A G}(M)$ is a triangle-free graph, then $\mathbb{S A G}(M)$ is a star graph.

Proof. Let $\mathbb{S A G}(M)$ be a triangle-free graph. By Lemma 3.7, $\mathbb{S A G}(M)$ contains no odd cycle. Now by [19, Proposition 1.6.1] $\mathbb{S A G}(M)$ is a bipartite graph. Suppose, on the contrary, $\mathbb{S} \mathbb{A}(M)$ is not star. Thus $\mathbb{S} \mathbb{G}(M)$ is a bipartite graph with parts $V_{1}, V_{2}$ such that $\left|V_{1}\right| \geq 2$ and $\left|V_{2}\right| \geq 2$. By Proposition 3.5, $\mathbb{A} \mathbb{G}(M)$ is also bipartite and hence $\mathbb{A} \mathbb{G}(M)$ is triangle-free. Now by [10, Theorem 2.13], $\mathbb{A} \mathbb{G}(M)$ is a star graph and so is $\mathbb{S} \mathbb{A}(M)$, a contradiction.

Corollary 3.17. Let $M$ be a finitely generated module, $\operatorname{ann}(M)$ a nil ideal and $|\operatorname{Min}(M)|=$ 1. If $\mathbb{S A G}(M)$ is a bipartite graph, then $\mathbb{S} \mathbb{G}(M)$ is a star graph.

Remark 3.18. Let u.dim $M=n$, where $M$ is an $R$-module. Then we have $U_{1} \oplus U_{2} \oplus$ $\ldots \oplus U_{n} \leq M$, where $U_{i} \neq 0$ for $1 \leq i \leq n$. By Lemma 2.4, $U_{i}$ and $U_{j}$ are adjacent for each $i \neq j$ and hence u.dim $M \leq \operatorname{cl}(\mathbb{S A G}(M))$.

Proposition 3.19. For every module $M, \operatorname{cl}(\mathbb{S} \mathbb{G}(M))=2$ if and only if $\chi(\mathbb{S A} \mathbb{G}(M))=2$; in particular $\mathbb{S} \mathbb{G}(M)$ is bipartite if and only if $\mathbb{S} \mathbb{G}(M)$ is triangle-free.

Proof. Suppose that $\operatorname{cl}(\mathbb{S A G}(M))=2$ and $\mathbb{S A G}(M)$ is not bipartite. Then $\mathbb{S A G}(M)$ contains an odd cycle and so by Lemma 3.7, the graph contains a triangle, a contradiction. Thus $\mathbb{S A G}(M)$ is bipartite and so $\chi(\mathbb{S A G}(M))=2$. The converse is clear.

If $M$ is a cyclic module, then clearly $M$ is a multiplication module and so by Proposition 2.3 , the two graphs $\mathbb{S} \mathbb{A}(M)$ and $\mathbb{A} \mathbb{G}(M)$ coincide. Now by [10], we have the following results.

Proposition 3.20. Let $M$ be a cyclic module.

(1) If $\left\{P_{1}, \cdots, P_{n}\right\}$ is a finite set of distinct minimal prime submodules of $M$, then $\mathbb{S A G}(M)$ has a clique of size $n$.

(2) $\operatorname{cl}(\mathbb{S A G}(M)) \geq|\operatorname{Min}(M)|$ and if $|\operatorname{Min}(M)| \geq 3$, then $\operatorname{gr}(\mathbb{S A G}(M))=3$.

(3) If $\operatorname{rad}_{M}(0)=(0)$, then $\chi(\mathbb{S A G}(M))=\operatorname{cl}(\mathbb{S A G}(M))=|\operatorname{Min}(M)|$.

Proposition 3.21. Let $M$ be a cyclic module and $\operatorname{ann}(M)$ be a nil ideal of $R$.

(1) If $\operatorname{rad}_{M}(0) \neq(0)$ and $|\operatorname{Min}(M)|=2$, then either $\mathbb{S A} \mathbb{G}(M)$ contains a cycle or $\mathbb{S A G}(M)=P_{4}$.

(2) If $|\operatorname{Min}(M)| \geq 3$, then $\mathbb{S} \mathbb{G}(M)$ contains a cycle.

\section{Dominating set and total dominating set}

For every graph $G$, the dominating number of $G$ and the total dominating number of $G$ are denoted by $\gamma(G)$ and $\gamma_{t}(G)$, respectively. A dominating set of cardinality $\gamma(G)\left(\gamma_{t}(G)\right)$ is called a $\gamma$-set $\left(\gamma_{t}\right.$-set). Several authors studied the (total) domination number in the 
zero-divisor graphs and the annihilating-ideal graphs, see for example $[20,22,23]$. In this section we compare $\gamma(\mathbb{S} \mathbb{G}(M))$ with $\gamma(\mathbb{A} \mathbb{G}(M))$ and also $\gamma_{t}(\mathbb{S} \mathbb{G}(M))$ with $\gamma_{t}(\mathbb{A} \mathbb{G}(M))$. Some results are similar to some of the results for $\gamma(\mathbb{A} \mathbb{G}(R))$ in [23]. In the following example we consider $M$ as a $\mathbb{Z}$-module. We remind that $V(\mathbb{A} \mathbb{G}(M))=V(\mathbb{S A G}(M))$.

Example 4.1. (1) If $M=\mathbb{Z}_{p} \oplus \mathbb{Z}_{p}$, where $p$ is prime, then $\gamma(\mathbb{S A G}(M))=1$ and $\gamma_{t}(\mathbb{S} \mathbb{A}(M))=2$, because $\mathbb{S} \mathbb{A} G(M)$ is a complete graph.

(2) If $M=\mathbb{Z}_{p} \oplus \mathbb{Z}_{p^{2}}$ and $N=\mathbb{Z}_{p} \oplus(0)$, then since $(N: M)=p^{2} \mathbb{Z}$, every vertex of $\mathbb{S A G}(M)$ is adjacent to $N$. Thus $\gamma(\mathbb{S A G}(M))=1$.

(3) If $M=\mathbb{Z}_{p} \oplus \mathbb{Z}_{q}$, where $p$ and $q$ are distinct primes, then $\gamma(\mathbb{S A G}(M))=1$ and $\gamma_{t}(\mathbb{S} \mathbb{A} \mathbb{G}(M))=2$.

(4) Let $M=\mathbb{Z}_{p q r}$, where $p, q, r$ are distinct primes. Then $\left\{N_{1}, N_{2}, N_{3}\right\}$ is both a $\gamma$-set and $\gamma_{t^{-}}$set, where $N_{1}=p q \mathbb{Z}_{p q r}, N_{2}=p r \mathbb{Z}_{p q r}$ and $N_{3}=q r \mathbb{Z}_{p q r}$. Thus $\gamma(\mathbb{S A G}(M))=$ $\gamma_{t}(\mathbb{S} \mathbb{A}(M))=3$.

(5) Let $M_{1}=M_{2}=\mathbb{Z}_{p}$ and $M=M_{1} \oplus M_{2}$. Then we have $\gamma(\mathbb{S A G}(M))=1$ and $\gamma\left(\mathbb{S A G}\left(M_{1}\right)\right)=\gamma\left(\mathbb{S A G}\left(M_{2}\right)\right)=0$. Hence $\gamma\left(\mathbb{S A G}\left(M_{1} \oplus M_{2}\right)\right) \neq \gamma\left(\mathbb{S A G}\left(M_{1}\right)\right)+\gamma\left(\mathbb{S A G}\left(M_{2}\right)\right)$.

Notice that for any $R$-module $M, \gamma(\mathbb{A G}(M)) \leq \gamma(\mathbb{S} \mathbb{G}(M))$. However, we have the following interesting result.

Theorem 4.2. Let $M$ be an R-module. Then

(1) $\gamma(\mathbb{S A G}(M))=1$ if and only if $\gamma(\mathbb{A} \mathbb{G}(M))=1$.

(2) If $\gamma(\mathbb{S A} \mathbb{G}(M))=2$, then $\gamma(\mathbb{A} \mathbb{G}(M))=2$.

(3) If $\gamma(\mathbb{A} \mathbb{G}(M))=n>1$, then $n \leq \gamma(\mathbb{S A G}(M)) \leq 2 n$

Proof. (1). Let $\gamma(\mathbb{S A G}(M))=1$ and $\{N\}$ be a $\gamma$-set. Then since every edge in $\mathbb{S A G}(M)$ is also an edge in $\mathbb{A} \mathbb{G}(M),\{N\}$ is a $\gamma$-set in $\mathbb{A} \mathbb{G}(M)$ and so $\gamma(\mathbb{S A} \mathbb{G}(M))=1$. Conversely, suppose that $\{N\}$ is a $\gamma$-set in $\mathbb{A} \mathbb{G}(M)$ and we set $N_{1}=M(N: M)$. If $N_{1}=0$, or $N_{1}=N$, then it is easy to see that $\{N\}$ is also a $\gamma$-set in $\mathbb{S A G}(M)$ and we are done. Now assume that $0 \neq N_{1} \subsetneq N$. Then for each vertex $K \neq N$ of $\mathbb{S} \mathbb{A} G(M)$, we have $M(N$ : $M)(K: M)=0$. Thus $N_{1}-N$ is an edge in $\mathbb{A} \mathbb{G}(M)$ and so $M\left(N_{1}: M\right)(N: M)=0$. We set $N_{2}=M\left(N_{1}: M\right)$. Again if $N_{2}=0$, then clearly $\left\{N_{1}\right\}$ is a $\gamma$-set in $\mathbb{S A G}(M)$. Now if $N_{2} \neq 0$, then we claim that $\left\{N_{2}\right\}$ is a $\gamma$-set in $\mathbb{S A G}(M)$. Since $N_{2}(N: M)=0$ and $N_{2} \neq N, N-N_{2}$ is an edge in $\mathbb{S A G}(M)$. Suppose that $K \neq N$ is another vertex of $\mathbb{S A G}(M)$. Then $K$ must be adjacent to $N$ in $\mathbb{A} \mathbb{G}(M)$ and so $M(N: M)(K: M)=0$. Since $N_{1} \subseteq N$, we have $N_{2}(K: M)=M\left(N_{1}: M\right)(K: M) \subseteq M(N: M)(K: M)=0$. Thus $K=N_{2}$ or $K$ is adjacent to $N_{2}$ in $\mathbb{S A} \mathbb{G}(M)$ and the proof is complete.

(2). Let $\gamma(\mathbb{S A} \mathbb{G}(M))=2$. Then $\gamma(\mathbb{A} \mathbb{G}(M)) \leq 2$. If $\gamma(\mathbb{A} \mathbb{G}(M))=1$, then by (1) we have $\gamma(\mathbb{S A} \mathbb{G}(M))=1$, a contradiction. Thus $\gamma(\mathbb{A} \mathbb{G}(M))=2$.

(3). Let $\gamma(\mathbb{A} \mathbb{G}(M))=n$ and $\left\{N_{1}, \cdots, N_{n}\right\}$ be a $\gamma$-set in $\mathbb{A} \mathbb{G}(M)$. We set $K_{i}=M\left(N_{i}: M\right)$ for $i=1,2, \cdots, n$. If $K_{i}=M\left(N_{i}: M\right)=0$, for some $i$, then $\left\{N_{i}\right\}$ is a $\gamma$-set in $\mathbb{A} \mathbb{G}(M)$ and hence $\gamma(\mathbb{A G}(M))=1$, a contradiction. We claim that $\left\{N_{1}, \cdots, N_{n}, K_{1}, \cdots, K_{n}\right\}$ is a dominating set in $\mathbb{S A G}(M)$. Let $K \notin\left\{N_{1}, \cdots, N_{n}\right\}$ be a vertex of $\mathbb{S A G}(M)$. Then $K$ is adjacent to $N_{i}$ in $\mathbb{A} \mathbb{G}(M)$ for some $i \in\{1, \cdots, n\}$. Thus $M\left(N_{i}: M\right)(K: M)=0$ and hence $K_{i}(K: M)=0$. This means that $K=K_{i}$ or $K$ is adjacent to $K_{i}$ in $\mathbb{S A G}(M)$. Thus we have $\gamma(\mathbb{S A} \mathbb{G}(M)) \leq 2 n$.

Proposition 4.3. For any $R$-module $M, \gamma_{t}(\mathbb{S} \mathbb{G}(M)) \leq \gamma_{t}(\mathbb{A} \mathbb{G}(M))$.

Proof. If $\gamma_{t}(\mathbb{A} G(M))=\infty$, there is no thing to prove. Let $\gamma_{t}(\mathbb{A} \mathbb{G}(M))=n$ and $\left\{N_{1}, \cdots, N_{n}\right\}$ be a $\gamma_{t}$-set in $\mathbb{A} \mathbb{G}(M)$. Set $K_{i}=M\left(N_{i}: M\right)$ for $i=1,2, \cdots, n$. If $K_{i}=0$ for some $i$, then $\left\{N_{i}, N\right\}$ is a $\gamma_{t}$-set in both the graph $\mathbb{A} \mathbb{G}(M)$ and the graph $\mathbb{S} \mathbb{G}(M)$, for every vertex $N \neq N_{i}$ and we are done. Now, suppose that $K_{i} \neq 0$ for every $1 \leq i \leq n$. We may assume the indexing is arranged such that $K_{1}, K_{2}, \cdots, K_{r}$ are pairwise distinct $(r \leq n)$. Let $K$ be a vertex in $\mathbb{S A G}(M)$. If $K=K_{i}$, for some $1 \leq i \leq n$, then since there 
exists $1 \leq j \leq n$ such that $N_{i}$ is adjacent to $N_{j}$ in $\mathbb{A} \mathbb{G}(M)$ we have $K_{i}$ is adjacent to $K_{j}$ in $\mathbb{S A G}(M)$. Now if $K \neq K_{i}$, for any $1 \leq i \leq n$, then there exists $1 \leq i \leq n$ such that $K$ is adjacent to $N_{i}$ in $\mathbb{A} \mathbb{G}(M)$. Thus $K_{i}(K: M)=M\left(N_{i}: M\right)(K: M)=0$ and so $K$ is adjacent to $K_{i}$. This means that $\left\{K_{1}, \cdots, K_{r}\right\}$ is a total dominating set in $\mathbb{S A G}(M)$. Therefore $\gamma_{t}(\mathbb{S A G}(M)) \leq n$.

Theorem 4.4. For any $R$-module $M, \gamma_{t}(\mathbb{S A G}(M))=\gamma(\mathbb{S A G}(M))$ or $\gamma_{t}(\mathbb{S A G}(M))=$ $\gamma(\mathbb{S A} \mathbb{G}(M))+1$.

Proof. Let $\gamma_{t}(\mathbb{S A G}(M)) \neq \gamma(\mathbb{S A G}(M))$ and $D$ be a $\gamma$-set in $\mathbb{S A G}(M)$. If $\gamma(\mathbb{S A G}(M))=1$, then it is clear that $\gamma_{t}(\mathbb{S} \mathbb{A}(M))=2$. So let $\gamma(\mathbb{S} \mathbb{A} G(M)) \geqslant 1$ and set $m=\max \{n \mid$ $\cap_{i=1}^{n} N_{i} \neq 0$, for some $\left.N_{1}, \cdots, N_{n} \in D\right\}$. Since $\gamma_{t}(\mathbb{S A G}(M)) \neq \gamma(\mathbb{S A} \mathbb{G}(M))$, we have $m \geq 2$. Suppose that $\cap_{i=1}^{m} N_{i} \neq 0$, for some $N_{1}, \cdots, N_{m} \in D$. Since $D$ is a $\gamma$-set in $\mathbb{S A G}(M)$, there exist distinct vertices $K_{1}, \cdots, K_{m}$ such that $K_{i}$ is adjacent to $N_{i}$ for $1 \leq i \leq m$. As $\cap_{i=1}^{m} N_{i} \subseteq N_{i}$, we conclude that $K_{i}$ is adjacent to $\cap_{i=1}^{m} N_{i}$, for each $i$. Now we claim that $S=\left\{\cap_{i=1}^{m} N_{i}, K_{1}, \cdots, K_{m}\right\} \cup D \backslash\left\{N_{1}, \cdots, N_{m}\right\}$ is a $\gamma_{t^{-}}$set in $\mathbb{S A G}(M)$. Let $L$ be a vertex of $\mathbb{S A G}(M)$. Then one of the following cases holds.

Case 1: $L \in D$. If $L \in\left\{N_{1}, \cdots, N_{m}\right\}$, then $L$ is adjacent to $K_{i}$, for some $1 \leq i \leq m$. If $L \notin\left\{N_{1}, \cdots, N_{m}\right\}$, then by the maximality of $m, \cap \cap_{i=1}^{m} N_{i} \cap L=0$ and hence $L$ is adjacent to $\cap_{i=1}^{m} N_{i}$.

Case 2: $L \notin D$. If $L$ is adjacent to one of the $N_{i}$ 's, then $L$ is adjacent to $\cap_{i=1}^{m} N_{i}$. Otherwise $L$ is adjacent to one of the element of $D \backslash\left\{N_{1}, \cdots, N_{m}\right\}$. This means that $S$ is a $\gamma_{t^{-}}$set in $\mathbb{S A G}(M)$. Thus $\gamma_{t}(\mathbb{S A G}(M))=\gamma(\mathbb{S A G}(M))+1$.

Example 4.5. (1) Let $R=\mathbb{Z}, M=\mathbb{Z}_{4}, N=2 \mathbb{Z}_{4}$. Then $\{N\}$ is both a $\gamma$-set and $\gamma_{t}$ set in $\mathbb{S A G}(M)$. Thus $\gamma_{t}(\mathbb{S} \mathbb{G}(M))=\gamma(\mathbb{S A G}(M))=1$.

(2) Let $R=\mathbb{Z}, M=\mathbb{Z}_{2} \oplus \mathbb{Z}_{2}, N_{1}=\mathbb{Z}_{2} \oplus(0)$, and $N_{2}=(0) \oplus \mathbb{Z}_{2}$. Then $\left\{N_{1}\right\}$ is a $\gamma$-set and $\left\{N_{1}, N_{2}\right\}$ is a $\gamma_{t^{-}}$set in $\mathbb{S A G}(M)$. Thus $\gamma(\mathbb{S A G}(M))=1$ and $\gamma_{t}(\mathbb{S} \mathbb{G}(M))=2$.

Acknowledgment. The authors are grateful to the referee for valuable suggestions towards improvement of the presentation.

\section{References}

[1] G. Aalipour, S. Akbari, M. Behboodi, R. Nikandish, M.J. Nikmehr and F. Shaveisi, The classification of the annihilating-ideal graphs of commutative rings, Algebra Colloq. 21 (2), 249-256, 2014.

[2] G. Aalipour, S. Akbari, R. Nikandish, M.J. Nikmehr and F. Shaveisi, On the coloring of the annihilating-ideal graph of a commutative ring, Discrete Math. 312, 2620-2626, 2012.

[3] S. Akbari, H.R. Maimani and S. Yassemi, When a zero-divisor graph is planar or a complete r-partite graph, J. Algebra, 270, 169-180, 2003.

[4] S. Akbari and A. Mohammadian, Zero-divisor graphs of non-commutative rings, J. Algebra, 296, 462-479, 2006.

[5] F. Aliniaeifard, M. Behboodi, E. Mehdi-nezhad and A.M. Rahimi, The annihilatingideal graph of a commutative ring with respect to an ideal, Comm. Algebra , 42, 2269-2284, 2014.

[6] D.F. Anderson, R. Levy and J. Shapiro J, Zero-divisor graphs, von Neumann regular rings, and Boolean algebras, J. Pure Appl. Algebra, 180, 221-241, 2003.

[7] D.F. Anderson and P.S. Livingston, The zero-divisor graph of a commutative ring, J. Algebra , 217, 434-447, 1999.

[8] D.D. Anderson and M. Naseer, Beck's coloring of a commutative ring, J. Algebra Appl. 159, 500-514, 1993. 
[9] H. Ansari-Toroghy and Sh. Habibi, The Zariski topology-graph of modules over commutative rings, Comm. Algebra 42, 3283-3296, 2014.

[10] H. Ansari-Toroghy and Sh. Habibi, The annihilating-submodule graph of modules over commutative rings II. Arab J. Math. 5, 187-194, 2016.

[11] H. Ansari-Toroghy and Sh. Habibi, The annihilating-submodule graph of modules over commutative rings, Math. Reports 20, 245-262, 2018.

[12] A. Azizi, Weakly prime submodule and prime submodule, Glasgow Math. J. 48, 343$346,2006$.

[13] I. Beck, Coloring of commutative rings, J. Algebra 116, 208-226, 1988.

[14] M. Behboodi and Z. Rakeei, The annihilating-ideal graph of commutative rings I, J. Algebra Appl. 10 (4), 727-739, 2011.

[15] R. Beyranvand and A. Farzi-Safarabadi, The strongly annihilating-submodule graph of a module, Algebraic Struc. Appl. 7 (1), 83-99, 2020.

[16] R. Beyranvand and F. Rastgoo, Weakly second modules over noncommutative rings, Hacet. J. Math. Stat. 45 (5), 1355-1366, 2016.

[17] S. Ceken, M. Alkan and P.F. Smith, Second modules over noncommutative rings, Comm. Algebra, 41, 83-98, 2013.

[18] F.R. DeMeyer, T. McKenzie and K. Schneider, The zero-divisor graph of a commutative semigroup, Semigroup Forum, 65, 206-214, 2002.

[19] R. Diestel, Graph Theory. Electronic Edition, New York: Springer-Verlag Heidelberg 1997, 2000, 2005.

[20] N. Jafari Rad, S.H. Jafari and D.A. Mojdeh, On domination in zero-divisor graphs, Canad. Math. Bull. 56 (2), 407-411, 2013.

[21] T.Y. Lam Lectures on modules and rings, Graduate Texts in Math. New York Heidelberg-Berlin: Springer-Verlag 1999.

[22] D.A. Mojdeh and A.M. Rahimi, Dominating sets of some graphs associated to commutative rings, Comm. Algebra, 40, 3389-3396, 2012.

[23] R. Nikandish, H. Maimani and S. Kiani, Domination numer in the annihilating-ideal graphs of commutative rings, Publications De L'institut Math. 97 (111), 225-231, 2015.

[24] S. Safaeeyan, Annihilating submodule graph for modules, Trans. Comb. 7 (1), 1-12, 2018.

[25] S. Safaeeyan, M. Baziar and E. Momtahan, A generalization of the zero-divisor graph for modules, J. Korean Math. Soc. 51 (1), 87-98, 2014.

[26] L. Toth, Subgroups of finite abelian groups having rank two via Goursats lemma, Tatra Mt. Math. Publ. 59 (1), 93-103, 2014. 